\title{
Influence of a future aerobic exercise session on pre-exercise energy intake: preliminary results
}

\author{
A. Barutcu, J. Moon, E. Briasco, G.L. Witcomb and L.J. James \\ School of Sport, Exercise and Health Sciences, Loughborough University, LE11 3TU, UK.
}

The effect of an acute aerobic exercise session on subsequent energy intake has been well investigated, with studies generally reporting little difference in post-exercise energy intake compared to a resting trial ${ }^{(1)}$. Despite this, little research has examined how an impending exercise session influences energy intake before the exercise session, which is surprising as exercise is rarely spontaneous, and exercisers may alter their energy or nutrient intake in anticipation of exercise. Therefore, this study examined energy and nutrient intake on the day before a fasted morning exercise session.

Six healthy, recreationally active males, who performed cardiovascular exercise $\geq 3$ times/ week (age 23 (6) y, BMI $24.2(2.0) \mathrm{kg} / \mathrm{m}^{2}$, body fat 17.7 (2.3) \%, $\mathrm{VO}_{2}$ peak $49.1(2.2) \mathrm{mL} / \mathrm{kg} / \mathrm{min}$ ) completed exercise (EX) and resting (REST) trials in a randomised, counterbalanced order, after completing a full familiarisation with the EX trial. On the morning of day 1, subjects attended the laboratory and were notified whether they would perform exercise or rest the following morning, before having access to buffet-style ad-libitum breakfast (08:00) and lunch (12:00) meals and being provided with an ad-libitum food bag for the evening (17:00-23:00). The next morning (day 2), subjects came to the laboratory at 08:00, and completed a 60 min exercise/ rest session in a fasted state. Thirty min after exercise/ rest, subjects were given access to an ad-libitum buffet breakfast. Subjective appetite sensations were measured periodically, with individual meal and $24 \mathrm{~h}$ energy intake quantified.

Energy expended during the 60 min exercise/ rest was greater during EX (EX 3103 (255) kJ; REST 319 (34) kJ; $P<0.05)$. Energy intake was similar between trials at breakfast on both days (day 1: EX 2931 (999) kJ; REST 2781 (856) kJ; $P=0.682$; day 2: EX 3184 $(1220) \mathrm{kJ}$; REST $2772(1348) \mathrm{kJ} ; P=0.458)$ and at lunch on day $1($ EX $2931(654) \mathrm{kJ}$; REST $2470(719) \mathrm{kJ} ; P=0.156)$. However, there was a trend for increased energy intake during EX for dinner (EX $6272(1759) \mathrm{kJ}$; REST $4889(1315) \mathrm{kJ} ; P=0.053)$ and the $24 \mathrm{~h}$ pre-exercise (EX 12133 (2735) kJ; REST 10139 (1948) kJ; $P=0.056)$, but relative energy intake (energy intake minus energy expended during the 60 min EX/ REST) was similar between trials (EX 9031 (2724) kJ; REST $9821(1935) \mathrm{kJ} ; P=0.383)$.

These preliminary results suggest that whilst exercisers do not appear to increase energy intake post-exercise, they might increase energy intake in anticipation of a future planned exercise session. This may explain some of the results seen by the chronic weight loss studies that report less than anticipated weight loss ${ }^{(2)}$.

1. Schubert MM, Desbrow B, Sabapathy S, et al. (2013) Appetite 63, 92-104

2. Turner JE, et al. (2010) Am J Clin Nutr 92, 1009-1016. 